\title{
RESPUESTA PRODUCTIVA DE VACAS LECHERAS EN PASTOREO AL MAÍZ FRESCO PICADO COMO SUPLEMENTO
}

\author{
PERFORMANCE RESPONSE OF GRAZING LACTATING DAIRY COWS TO CHOPPED \\ FRESHMAIZE AS SUPPLEMENT
}

\author{
Ramírez, M.M. ${ }^{1}$, Hernández, O. ${ }^{1 *}$, Améndola, R.D. ${ }^{2}$, Mendoza, G.D. ${ }^{1,3}$, Ramírez, E.J. ${ }^{1}$ \\ y Burgueño, J.A. ${ }^{4}$
}

${ }^{1}$ Colegio de Postgraduados. Municipio de Texcoco. México. *ohmendo@colpos.mx ${ }^{2}$ Departamento de Zootecnia. Universidad Autónoma Chapingo. Municipio de Texcoco. México. ${ }^{3}$ Departamento de Producción Agrícola y Animal. Universidad Autónoma Metropolitana Unidad Xochimilco. Delegación Coyoacán. México D.F.

${ }^{4}$ Centro Internacional de Mejoramiento del Maíz y Trigo. El Batán. Municipio de Texcoco. México.

\section{PALABRAS CLAVE ADICIONALES}

Consumo de materia seca. Carga animal.

\section{RESUMEN}

A 24 vacas Holstein multíparas en pastoreo rotacional en una pradera mixta alfalfa-pasto oviIlo, se suministraron: 0,4 y $8 \mathrm{~kg}$ de MS de maíz fresco picado (MFP) animal-1 día ${ }^{-1}$. Además, cada animal recibió $3,8 \mathrm{~kg}$ de $\mathrm{MS}$ de alimento concentrado. Se utilizó un diseño reversible simple (crossover) (tres tratamientos, dos repeticiones con cuatro animales cada uno), de 3 períodos, con una duración de 19 días cada uno (10d de adaptación y $9 \mathrm{~d}$ de muestreo). No hubo diferencias significativas en producción individual ni en composición de la leche. El consumo de pradera disminuyó $(p<0,0001)$ de 8,4 a 5,2 y 3,1 kg de MS de pradera al aumentar el nivel de MFP de 0 a 4 y $8 \mathrm{~kg}$ de MS, respectivamente; mientras que el consumo total de MS incrementó $(p<0,0001)$ en aquellas vacas con un nivel de suplemento más alto, promediando $12,2,12,9$, y 14,8 kg de MS total con 0,4 y $8 \mathrm{~kg}$ de MS de MFP, respectivamente. La tasa de sustitución fue de 0,75 y 0,67 kg de MFP con 4 y $8 \mathrm{~kg}$ de MS de MFP respectivamente, consecuentemente incrementó $(p<0,0001)$ la carga animal $(C A)$, de 3,8 a 6,2, y 8,8 vacas ha-1 al aumentar de 0 a 4 y $8 \mathrm{~kg}$ de MS de MFP, respectivamente, mientras que la producción de leche por hectárea incrementó ( $p<0,0001)$ de 39,3 a 64,5 y 95,5 kg de leche, respectivamente. Esto indica que por cada $\mathrm{kg}$ de

\section{AdditionAL KEYWORD}

Dry matter intake. Stocking rate.

MS de MFP, la CA aumenta 0,63 vacas y $0,75 \mathrm{~kg}$ de leche ha- ${ }^{-1}$. La suplementación con MFP a vacas en etapa final de lactancia no presentó beneficios en producción de leche individual, ni cambios en composición de la leche, pero sí incrementó la producción de leche por hectárea, como resultado de aumento en la CA.

\section{SUMMARY}

Twenty four multiparous Holstein dairy cows were rotationally grazed on a mixed alfalfaorchard sward. Three treatments were evaluated: 0,4 , and $8 \mathrm{~kg}$ of DM of MFP $\operatorname{cow}^{-1}$ day $^{-1}$. Each cow received $3.8 \mathrm{~kg}$ of concentrate (DM basis). The experimental design was a cross-over (3 treatments, 2 replicates with 4 animals each), with 3 periods lasting 19 days each (10 adaptation plus 9 sampling period). There were no differences on individual milk production nor on milk composition. Herbage dry matter intake decreased $(p<0.0001)$ from 8.4 to 5.2 and $3.1 \mathrm{~kg}$ of DM when increasing MFP from 0 to 4 and $8 \mathrm{~kg}$ of DM, respectively; while total DM intake increased $(p<0.0001)$ on those cows with the highest level of supplementation, averaging 12.2, $12.9,14.8 \mathrm{~kg}$ of total DM at 0,4 , and $8 \mathrm{~kg}$ of DM of 


\section{RAMÍREZ, HERNÁNDEZ, AMÉNDOLA, MENDOZA, RAMÍREZY BURGUEÑO}

FCM, respectively. Substitution rate of pasture for MFP was 0.75 and $0.67 \mathrm{~kg}$ of pasture per $\mathrm{kg}$ of $\mathrm{FCM}$ with 4 and $8 \mathrm{~kg}$ of DM of MFP, respective. Consequently, stocking rate (STR) increased $(p<0.0001)$ from 3.8, to 6.2, and 8.8 cows ha- ${ }^{-1}$, with 0,4 , and $8 \mathrm{~kg}$ of DM of MFP, respectively; and milk production per hectare increased $(p<0.0001)$ from 39.3 to 64.5 and $95.5 \mathrm{~kg}$ of milk with 0,4 , and $8 \mathrm{~kg}$ of DM of FCM, respectively. This means that STR increases 0.63 cows ha-1, and $0.75 \mathrm{~kg}$ of milk when MFP is increased in one $\mathrm{kg}$ of DM. It is suggested that offering MFP as supplement to grazing dairy cows in their final lactation stage had no benefits in individual milk production or milk composition; but on milk production per hectare.

\section{INTRODUCCIÓN}

La producción de leche en estabulación, tiene costos muy altos comparados con los sistemas de producción basados en pastoreo. Sin embargo, estos enfrentan el problema del bajo consumo de MS, que si es de baja calidad disminuye la producción. La suplementación podría aliviar este problema e incrementar la producción de leche, ya que genera un efecto sustitutivo, por lo que se incrementa la carga animal y la producción por hectárea. Fike et al. (2003) demostraron que al aumentar la carga animal de 7,5 a 10 vacas, la producción de leche aumenta de 82 a $119 \mathrm{~kg} \mathrm{ha}^{-1}$ si se suplementa con concentrado. Este beneficio está en función de la cantidad y calidad de forraje disponible (Sollenberger et al., 2005), así como de la cantidad y tipo de suplemento ofrecido (Bargo et al., 2003).

De los alimentos más comunes que se ofrecen como suplemento a vacas productoras de leche está el maíz ensilado (Bargo et al., 2003). Aunque su respuesta varía; así, Hernandez-Mendo y Leaver (2004-2006) reportaron solo efectos benéficos cuando los animales pastorearon a menor altura de forraje, cuando la disponibilidad de forraje fue baja y no recibieron ningún otro suplemento mientras que Holden et al. (1995) no encontraron efecto en producción ni en composición de la leche al ofrecer $2,3 \mathrm{~kg}$ de
MS de ensilado de maíz a vacas altas productoras, Améndola (2002) reportó que la producción de leche de vacas en pastoreo sin ensilado de maíz fue mayor $(19,5 \mathrm{~kg}$ vaca $^{-1}$ día $\left.^{-1}\right)$, que aquellas con $4,8 \mathrm{~kg}$ de materia seca de ensilado de maíz $(17,6 \mathrm{~kg}$ vaca $^{-1}$ día $^{-1}$ ), debido, probablemente, a la superior calidad y abundancia del alimento en la pradera. Nos obstante la suplementación con maíz ensilado permite incrementar la carga animal dado el efecto sustitutivo. Améndola (2002) reporta que la carga animal puede incrementarse de 2,1 a 3,6 vacas ha $^{-1}$, con 0 y 4,8 $\mathrm{kg}$ de MS de ensilado de maíz vaca ${ }^{-1}$ día $^{-1}$, aumentando, en consecuencia, la producción de leche por ha a una tasa de $0,79 \mathrm{~kg}$ extra de leche por cada $\mathrm{kg}$ de materia seca de ensilado de maíz ofrecido a pesar de que la producción individual se redujo con el ensilado. Debido a una alta tasa de sustitución, $(1,36)$ de la MS del pasto por la del ensilado; valor superior al obtenido $(0,11$ a 0,5$)$ con granos (Bargo et al., 2003).

La suplementación con maíz ensilado, precisa infraestructura de ensilaje, por lo que utilizar maíz fresco picado como suplemento podría ser mejor; hay poca o nula investigación que discuta este aspecto, a pesar de que en algunas explotaciones es una práctica común, porque, además conserva más nutrientes que otros métodos, como la henificación o ensilaje (Church et al., 2003; Darby y Lauer, 2002). El objetivo de este estudio fue evaluar la respuesta productiva de vacas en lactancia pastoreando en una pradera mixta de alfalfa-pasto ovillo con un suplemento de maíz fresco picado.

\section{MATERIALES YMÉTODOS}

El estudio se llevó a cabo del 7 de agosto al 25 de octubre de 2006 en el módulo de producción de leche en pastoreo de la Universidad Autónoma Chapingo, en el estado de México, localizado a $19^{\circ} 29^{\prime} \mathrm{N}, 98^{\circ} 54^{\prime} \mathrm{O}$, y a $2240 \mathrm{~m}$ sobre el nivel del mar. El clima es 


\section{MAÍZ FRESCO PICADO COMO SUPLEMENTO A VACAS LECHERAS}

templado subhúmedo, con lluvias en verano; $1620 \mathrm{~mm}$ de precipitación y temperatura promedio de $18^{\circ} \mathrm{C}$ (García, 1988).

Se utilizaron 24 vacas Holstein multíparas en lactancia con valor inicial promedio de $508 \pm 259$ días en leche, $651 \pm 53 \mathrm{~kg}$ de peso vivo, $12,9 \pm 4,1 \mathrm{~kg}$ de leche, $7,6 \pm 1,7$ años de edad y $5,3 \pm 1,6$ partos. Se utilizó un diseño cross-over (Conchran et al., 1941), con 3 tratamientos y 2 repeticiones (lotes), y 4 vacas en cada uno, en 3 períodos, con una duración de 19 días cada uno (10 días de adaptación y 9 días de toma de muestras). Todas las vacas tuvieron una etapa de 14 días de adaptación al maíz fresco picado. Los animales fueron distribuidos homogéneamente en 6 grupos de 4 vacas cada uno, de acuerdo a la producción de leche. Se utilizaron 6 ha de pradera, divididas en 12 potreros, subdivididos en 6 subpotreros cada uno ( 3 orientados al norte y 3 al sur), y fueron pastoreados por cada uno de los 6 grupos de vacas previamente asignado de manera aleatoria, de tal modo que los tres tratamientos estuvieran presentes en los subpotreros del norte y sur. Posteriormente, los 6 grupos fueron asignados aleatoriamente a cada uno de tres tratamientos en estudio, que fueron 0,4 y $8 \mathrm{~kg}$ de MS de MFP animal ${ }^{-1}$ día $^{-1}$. Durante el período experimental, el promedio de MS del MFP fue de $28 \pm 4,7 \%$. El pastoreo fue rotacional en una pradera mixta de alfalfa (Medicago sativa) y pasto ovillo (Dactylis glomerata), con agua a libre acceso. Las vacas que no recibieron MFP, regresaron al potrero inmediatamente después de la ordeña, la cual se realizó dos veces al día, de las 6:00 a 8:30 y de las 14:00 a 16:00 horas. Diariamente se registró la hora de llegada a la sala de ordeña y la hora de salida de las corraletas de cada grupo de vacas, para determinar el tiempo en la pradera de cada una de ellos.

Se ofreció pasto fresco, por lo menos dos veces al día, usando un cerco eléctrico movible, de acuerdo con una estimación visual de la cantidad residual. Todas las vacas pastorearon el mismo potrero a un mismo tiempo y, cuando el forraje se agotaba en alguno de los subpotreros, los 6 grupos de vacas se movían a un potrero nuevo.

El maíz utilizado fue la variedad San José. Las fechas de siembra fueron: 24 de abril, 2, 8 y 22 de mayo, y 12 de junio de 2006, con el objetivo de mantener constante su estado de madurez al momento de ofrecerlo a los animales. El maíz se cortó y picó diariamente antes de la ordeña de la mañana con la ayuda de un tractor de tiro y una picadora. La cantidad de maíz fresco ofrecida dependía de su contenido de materia seca, la cual se obtenía cada tercer día después de ser cortado y picado, utilizando un horno de microondas, después de ciclos consecutivos de 4 minutos cada uno. La cantidad de maíz total diaria se ofreció, en grupo, dos veces al día, la mitad después de cada ordeña, con base en el tratamiento asignado.

El concentrado (tabla I y II) se formuló considerando la cantidad de MFP ofrecido y el consumo estimado en los potreros, de tal modo que las dietas totales (MFP+pradera+concentrado) fueran isoproteicas (17\% de PB).

La determinación del pasto ofrecido y residual se realizó un día antes y un día después de la ocupación del potrero, usando una podadora de pasto, se cosechó forraje en dos franjas de aproximadamente $8 \times 0,52 \mathrm{~m}$ en cada subpotrero de pastoreo, a una altura aproximada de $6 \mathrm{~cm}$. El pasto segado se pesó en fresco y una muestra se desecó en estufa a $60^{\circ} \mathrm{C}$ durante 48 horas y se guardó para análisis. Antes de cosechar el forraje, se registraron 10 alturas del forraje previamente identificado, usando un disco de $0,5 \mathrm{~m}^{2}$. Ya cortada la franja, se colocó un cuadro de metal de $80 \times 40 \mathrm{~cm}$ sobre la franja ya podada y se cortó a ras de suelo el forraje residual dejado por la podadora. Todo el forraje de tal área fue guardado, se pesó y se secó en estufa a $60^{\circ} \mathrm{C}$ por $48 \mathrm{~h}$ para su análisis.

Se tomaron muestras y mediciones en los ocho potreros pastoreados en los tres períodos experimentales (tres potreros en 
cada uno de los períodos 1 y 3 , y dos en el período 2). Un día antes del inicio de cada periodo, se tomaron muestras de pasto de cada potrero mediante pastoreo simulado que fueron secadas en estufa a $60^{\circ} \mathrm{C}$ por 48 h y guardadas para análisis.

El consumo de forraje se determinó mediante marcadores, utilizando óxido de cromo $\left(\mathrm{Cr}_{2} \mathrm{O}_{3}\right)$ como marcador externo y cenizas insolubles en ácido (CIA) como marcador interno (Geerken et al., 1987). Durante los tres períodos experimentales, los animales recibieron $16 \mathrm{~g}$ de óxido de cromo en dos tomas de $8 \mathrm{~g}$, en el alimento concentrado, durante la ordeña. Se tomaron muestras de heces en cuatro de los nueve días de obtención de muestras, en días alternos, directamente del recto del animal, que se colocaron en bolsas de polipapel y se congelaron para análisis. Descongeladas las muestras a temperatura ambiente se mezclaron las de cada vaca y período, para formar una sola compuesta, la cual fue analizada. A las heces y a los componentes de las dietas (pradera, concentrado y maíz fresco picado) se les determinaron cenizas insolubles en ácido. Aplicando la fórmula descrita por Church (1988), se calculó la producción diaria de materia fecal, y finalmente el consumo individual de materia seca mediante la formula descrita por Geerken et al. (1987):

PTH= Producción total de heces MS $\left(\mathrm{g} \mathrm{d}^{-1}\right)=$ Dosis del marcador $\left(\mathrm{g} \mathrm{d}^{-1}\right) /$ concentración del marcador en heces $\left(\mathrm{gg}^{-1} \mathrm{MS}\right)$.

El consumo de pradera $\left(\mathrm{g} \mathrm{MS} \mathrm{d}^{-1}\right)$ de cada vaca se estimó con la fórmula de Geerken et al. (1987):

Consumo de $\mathrm{MS}\left(\mathrm{g} \mathrm{d}^{-1}\right)=\left[\left(\mathrm{ClA}_{\mathrm{H}}{ }^{*} \mathrm{PTH}\right)-\left(\mathrm{ClA}{ }_{\mathrm{C}}{ }^{*} \mathrm{CTC}\right)-\right.$ $\left.\left(\mathrm{CIA}_{\mathrm{M}}{ }^{*} \mathrm{CTM}\right)\right] / \mathrm{CIA}$

Donde:

$\mathrm{ClA}_{\mathrm{H}}=$ Concentración de cenizas insolubles en ácido (CIA) en heces $\left(\mathrm{g} \mathrm{kg}^{-1} \mathrm{MS}\right)$;

PTH= Producción total de heces;

$\mathrm{CIA}_{\mathrm{C}}=$ Concentración de CIA del concentrado ( $\mathrm{g}$ $\mathrm{kg}^{-1} \mathrm{MS}$ );

$\mathrm{CTC}=$ Consumo total del concentrado $(\mathrm{g})$;

$\mathrm{CIA}_{\mathrm{M}}=$ Concentración de CIA en el maíz picado fresco $\left(\mathrm{g} \mathrm{kg}^{-1} \mathrm{MS}\right)$;

CTM = Consumo total de maíz picado fresco $\left(\mathrm{g} \mathrm{d}^{-1}\right)$;

$\mathrm{CIA}_{\mathrm{P}}=$ Concentración de CIA en el forraje de la pradera ( $\left.\mathrm{g} \mathrm{g}^{-1} \mathrm{MS}\right)$.

La tasa de sustitución se estimó a partir de una regresión lineal entre el consumo de pasto y el consumo de maíz fresco picado (Améndola, 2002). La carga animal y la pro-

Tabla I. Ingredientes del concentrado de acuerdo al tratamiento. (Ingredients of the concentrate according to treatment).

\begin{tabular}{|c|c|c|c|}
\hline & \multicolumn{3}{|c|}{ Maíz fresco picado (kg MS animal-11ia-1) } \\
\hline & 0 & 4 & 8 \\
\hline Sorgo molido & 46,47 & 28,5 & 4,54 \\
\hline Maíz molido & 45,79 & 24,94 & 3,82 \\
\hline Pasta de soya & 0 & 36,82 & 80,01 \\
\hline Melaza & 4,74 & 4,75 & 4,78 \\
\hline Ortofosfato de calcio & 1,78 & 0 & 0 \\
\hline Carbonato de calcio & 0 & 4,04 & $5,90 \mathrm{gg}$ \\
\hline $\mathrm{Sal}^{1}$ & 0,95 & 0,95 & 0,96 \\
\hline
\end{tabular}

Archivos de zootecnia vol. 60, núm.231, p. 650. 


\section{MAÍZ FRESCO PICADO COMO SUPLEMENTO A VACAS LECHERAS}

ducción de leche por hectárea se determinaron de acuerdo con lo descrito por Améndola (2002).

La producción de leche se midió individualmente utilizando una báscula (Truetest, capacidad de 1,5 ton), en cinco de los nueve días de muestreo, justo después de cada ordeña. La composición de la leche (porcentaje de grasa, proteína y lactosa) se analizó utilizando un Milkoscan, en el laboratorio de leches de la Universidad Autónoma Metropolitana, Unidad Xochimilco. Las muestras se colectaron en los mismos días que se midió la producción de leche, en ambas ordeñas. Se colocaron en frascos de plástico $(120 \mathrm{ml})$ y se mantuvieron en congelación hasta el día de su análisis, cuando se descongelaron a baño maría. El resultado del análisis se ajustó con base en la producción de leche correspondiente.

El análisis químico del pasto, MFP, concentrado y heces se realizó en el laboratorio de nutrición animal del programa de ganadería del Colegio de Postgraduados. A las muestras de MFP, pradera (pastoreo simu- lado) y alimento concentrado se les determinó: materia seca (AOAC, 1984), fibra detergente neutro (FDN) y ácido (FDA) (Goering y Van Soest, 1970), proteína por el método de microkjeldahl y cenizas (AOAC, 1984) y digestibilidad in vitro (Tilley y Terry, 1963). A las muestras de la pradera (ofrecido y residual) obtenidas con la podadora se le determinó únicamente cenizas, y a las de heces, materia seca (MS) y cenizas (AOAC, 1984) y también cromo, mediante absorción atómica (Williams et al., 1962). A todas las muestras, excepto las obtenidas con la podadora, se les determinaró CIA (Geerken et al.,1987).

Las vacas se pesaron al inicio y al final de cada período experimental (cada 19 d). El peso vivo final de un período se utilizó como peso vivo inicial del siguiente. Al término de la investigación, a todas las vacas se les realizó un diagnóstico de gestación por medio de ultrasonido (Sonovet 600, Universal Medical System, Inc. Transductor de 7,5 Mhz).

Los datos se sometieron a un análisis de

Tabla II. Composición química de la pradera, maíz fresco picado (MFP), concentrado y dieta total para cada tratamiento. (Chemical composition of forage (simulated grazing), corn, chopped fresh, focused and total diet for each treatment).

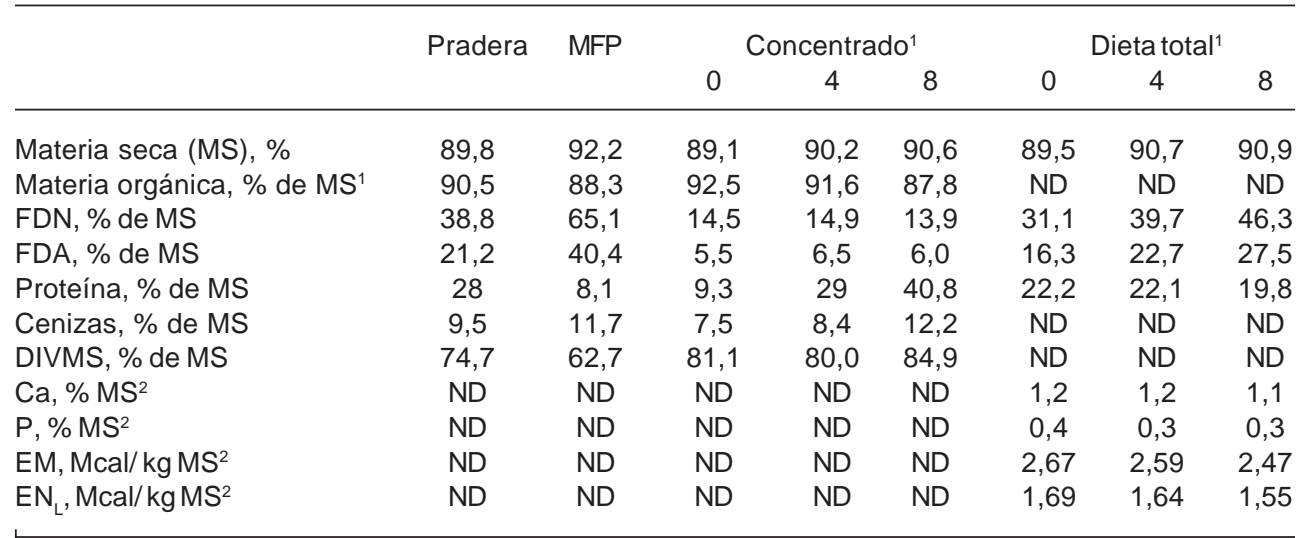

FDN: fibra detergente neutra; FDA: fibra detergente ácida; DIVMS: digestibilidad in vitro de la materia

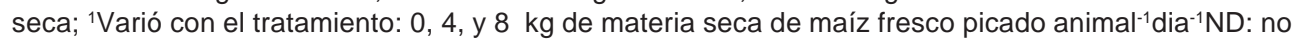
determinado; '2Estimados a partir de datos del NRC para ganado lechero (2001); $\mathrm{EN}_{\mathrm{L}}=$ Energía neta de lactancia. 
varianza, y el modelo estadístico usado fue:

$$
Y_{i j k}=\mu+P_{i}+T_{j}+(P \times T)_{i j}+E_{i j k}
$$

Donde:

$Y_{i j k}=$ variable de respuesta;

$\mu=$ media general;

$\mathrm{P}_{\mathrm{i}}=$ efecto del período;

$\mathrm{i}=3$;

$\mathrm{T}_{\mathrm{i}}=$ efecto del tratamiento;

$\mathrm{j}=3$;

$(\mathrm{PxT})_{\mathrm{ij}}=$ efecto de la interacción $\mathrm{x}$ período tratamiento;

$E_{i \mathrm{jk}}=$ error experimental.

Las variables de respuesta analizadas fueron: producción de leche individual y por hectárea, composición de la leche, consumo de MS total, consumo de forraje de pradera, tasa de sustitución y carga animal. Los resultados fueron analizados utilizando el procedimiento MIXED de SAS versión 8 (1999). El peso vivo inicial se usó como covariable en la producción de leche individual y la composición de la leche.

\section{RESULTADOSYDISCUSIÓN}

\section{CONSUMO DE MATERIA SECA Y TASA DE SUSTITUCIÓN}

El consumo de la pradera y el total de materia seca fueron afectados con el nivel de maíz fresco picado; en la tabla III se observa que el consumo de pradera disminuyó 38,9 y $62,6 \%(\mathrm{p}<0,0001)$ al aumentar el nivel de maíz fresco picado a 4 y $8 \mathrm{~kg}$, respectivamente; mientras que el consumo total de materia seca fue mayor en 22,2 y $15,4 \%$ en aquellas vacas con un nivel de suplemento de $8 \mathrm{~kg}$ vs. 0 y $4 \mathrm{~kg}$ de MS de MFP, respectivamente $(\mathrm{p}<0,0001)$. No obstante, esto no se reflejó en un incremento significativo de la producción de leche o en peso vivo, y puede deberse al tipo de ingredientes de cada uno de los concentrados. Por ejemplo, a diferencia del tratamiento sin MFP, los tratamientos con 4 y $8 \mathrm{~kg}$ de mate- ria seca de MFP vaca-1 $\mathrm{d}^{-1}$, para igualar el porcentaje de proteína, incluían pasta de soya que, debido a su alto porcentaje de proteína, estimula el consumo de materia seca (Mulligan et al., 2004).

Además, la fracción de FDN, en el consumo de materia seca (NRC, 2001), no fue igual en los diferentes tratamientos, incrementándose al aumentar la cantidad de MFP ofrecido a las vacas $(31,1,39,7, y$ 46,3 $\%$ de FDN de las dietas con 0,4, y $8 \mathrm{~kg}$ de MS de MFP vaca-1 $\mathrm{d}^{-1}$ ), pero no afectó negativamente el consumo total de materia seca. Contrariamente, Ruiz et al. (1995) indicaron que el consumo de materia seca y la producción de leche disminuyen linealmente cuando la FDN de la dieta se incrementa de 31 a 39\% (las dietas con MFP aquí ofrecidas contenían más de $39 \%$ de FDN). Mertens (1985; citado por Vazquez y Smith, 2000) establece que el consumo de materia seca se relaciona con la capacidad de consumo de FDN, la cual es de alrededor del 1,2\% del peso vivo del animal, aunque Vazquez y Smith (2000) reportan que puede ser hasta de $1,78 \%$ del peso vivo, cuando se ofrece un suplemento de forraje a vacas en pastoreo. En esta investigación, el consumo de FDN, como porcentaje del PV, fue menor a 1,05\%; por lo que otros factores, como los que aquí se mencionan, afectaron el consumo total de materia seca.

El consumo total de MS es similar a lo encontrado por otros autores (Vazquez y Smith, 2000). Sin embargo, aun cuando el consumo total de materia seca fue mayor con el suplemento de maíz, éste es menor a lo reportado por Améndola (2002), para el mismo tipo de animales y condiciones, con ensilado de maíz. Este autor encontró consumo total de materia seca de $15,9 v s .14,4 \mathrm{~kg}$ vaca $^{-1} \mathrm{~d}^{-1}, \mathrm{y}$ de forraje de pradera de 13,6 vs. $8,2 \mathrm{~kg}$ vaca $^{-1} \mathrm{~d}^{-1}$ con 0 o $4 \mathrm{~kg}$ de MS vaca $\mathrm{d}^{-1} \mathrm{~d}^{-1}$, respectivamente. Es posible que el nivel de producción de leche de las vacas experimentales pudo influir en el bajo consumo total de materia seca, si consideramos que la producción de leche promedio fue de $10 \mathrm{~kg}$ 


\section{MAÍZ FRESCO PICADO COMO SUPLEMENTO A VACAS LECHERAS}

vaca $^{-1}$ día $^{-1}$, como reportaron HernándezMendo y Leaver (2006), quienes encontraron un consumo total de materia seca de 18,3 vs. $14,9 \mathrm{~kg} \mathrm{vaca}^{-1} \mathrm{~d}^{-1}$ para vacas en pastoreo con 34,5 y $22 \mathrm{~kg}$ de leche vaca $^{-1} \mathrm{~d}^{-1}$. El aumento del consumo total de materia seca y disminución del consumo de pradera, es indicativo de un efecto sustitutivo que, en este estudio, fue de $0,67 \mathrm{~kg}$ de materia seca de pradera por cada $\mathrm{kg}$ de materia seca de MFP. El tipo de suplemento influye en la tasa de sustitución, y que los forrajes, como el MFP, disminuyen el consumo de pradera más que los concentrados. Con ensilado de maíz se reporta una tasa de sustitución de 0,84 a 1,02 (Bargo et al., 2003) hasta 1, 2 a $1,36 \mathrm{~kg}$ de materia seca de pradera por materia seca de ensilado de maíz (Holden et al., 1995; Améndola, 2002). Estos valores son superiores a lo que se menciona en esta investigación, pero mayor que lo reportado para un suplemento concentrado, que va desde 0,11 (Bargo et al., 2003) hasta 0,55 (Bargo et al., 2002) kg de MS de pradera por $\mathrm{kg}$ de MS de concentrado. Sin embargo, no siempre la tasa de sustitución es mayor con los suplementos a base de forrajes, pues se han reportado cifras similares usando suplementos tanto de heno como de grano (Bargo et al., 2003). La tasa de sustitución, es mayor cuando la disponibilidad del forraje es alta, como describen Bargo et al. (2002), quienes mencionan para baja disponibilidad de forraje (de 7,6 a 22,2 $\mathrm{kg}_{\text {de }} \mathrm{MS} \mathrm{vaca}^{-1}$ día $\left.^{-1}\right)$, una tasa de sustitución media de 0,19 $\mathrm{kg}$ de MS de pradera por $\mathrm{kg}$ de MS de concentrado; mientras que cuando la disponibilidad es alta (de 24 a $42,3 \mathrm{~kg}$ de MS vaca $^{-1}$ día $^{-1}$ ), la tasa de sustitución es de 0,58 $\mathrm{kg}$ de MS de pradera por $\mathrm{kg}$ de materia seca de concentrado, valores similares a los de este estudio, en que la disponibilidad de la pradera fue, en promedio, de 33,9 $\pm 1,99 \mathrm{~kg}$ de MS vaca-1 día $^{-1}$, y la tasa de sustitución de $0,67 \mathrm{~kg}$ de MS de pradera por kg de materia seca de MFP. Otro factor que influye en la respuesta a la suplementación, y en consecuencia en la tasa de sustitución, es el estado de la lactancia, ya que en etapas tempranas de ésta, el animal destina más nutrientes, tanto del alimento como de sus reservas corporales, para producción de leche; mientras que la lactancia tardía se destinan para mejorar la condición corporal (Kellaway y Harrington, 2004), por ello, la tasa de sustitución es menor en vacas en lactancia temprana $(0,13)$ que el de aquéllas en etapas medias o tardías $(0,49$ y 0,41 , respetivamente) (Penno et al., 2006).

\section{Producción de LECHE INDIVIDUAL Y COM- POSICIÓN DE LA LECHE}

La producción individual y composición de la leche se presentan en la tabla III. No hubo diferencias significativas entre tratamientos, resultados que concuerdan con otros en los que se ofreció ensilado de maíz a vacas en pastoreo (Fike et al., 2002; Holden et al., 1995). El nivel de producción de leche en esta investigación se encuentra por debajo de lo reportado en la mayoría de los trabajos de vacas en pastoreo (Bargo et al., 2002), que es de 19,1 kg (sin ningún suplemento), hasta $29,9 \mathrm{~kg} \mathrm{vaca}^{-1} \mathrm{~d}^{-1}$ (con suplemento concentrado). Esto puede deberse, parcialmente, a los días en lactancia de las vacas al inicio del experimento de 508 días, en promedio; por lo que, de acuerdo a Cumminghan (1999) se encuentra bien sobrepasado el pico de lactación y por tanto en fase avanzada de descenso. Dicho comportamiento se observó en este estudio, al disminuir $(\mathrm{p}<0,05)$ la producción de leche de 11,85 a $8,75 \mathrm{~kg}$ de leche por vaca-1 día $^{-1}$, del primer al tercer periodo de evaluación.

Según Bargo et al. (2003) en la etapa tardía de lactancia el efecto de un suplemento concentrado en la producción de leche es nulo, ya que se destinan a ganancia de peso, situación que pudo estar ocurriendo al ofrecer MFP a las vacas experimentales, cuyo estado de lactancia era muy avanzado, mientras que las vacas de los estudios de Delahoy et al., 2003; Holden et al., 1995 y Kolvert y Muller, 1998 tenían de 59 a 216 días en lactancia al inicio del experimento. 


\section{RAMÍREZ, HERNÁNDEZ, AMÉNDOLA, MENDOZA, RAMÍREZY BURGUEÑO}

Por otra parte, es importante mencionar que el $42 \%$ de las vacas aquí utilizadas tenían más de 4 meses de gestación al final del periodo experimental, que unido a lo anterior, pudo influir en su baja producción láctea, pues destina los nutrientes para la formación y crecimiento del feto, siendo de

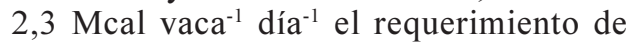
energía fetal en una vaca Holstein con 250 días de gestación (Ingvartsen y Andersen, 2000). Roche (2003) menciona que la producción de leche disminuye $0,8 \mathrm{~kg} \mathrm{vaca}^{-1} \mathrm{~d}^{-1}$ después de 147 días de gestación y 33 semanas en lactancia. Esto puede deberse a un tipo de regulación en el reparto de nutrientes denominado homeoresis, (cambios metabólicos coordinados para apoyar un estado fisiológico), que en este caso es la gestación, período durante el que los nutrientes maternos son para el desarrollo del feto, crecimiento de la membranas fetales, útero grávido, y glándula mamaria, especialmente durante el último tercio de la gestación y, en su caso, al inicio de la lactancia (Ingvartsen y Andersen, 2000).

El porcentaje de grasa, proteína, y lactosa encontrado en esta investigación tampoco varió $(\mathrm{p}>0,05)$. Resultados similares han sido reportados en otras investigaciones llevadas a cabo en pastoreo y con un suplemento a base de grano de maíz (Pulido et al., 2002; Wilkerson et al., 1997), y con ensilado de maíz (Améndola, 2002; Holden et al., 1995). Esto indica que la calidad de la leche no es afectada por suplementar con MFP.

Es interesante observar que a pesar de la diferencia en el consumo de materia seca (tabla III), la respuesta en producción de leche fue nula, aunque con mayor cantidad de MFP en la dieta, el consumo de energía incrementó de 37,5 y 38,9 Mcal vaca ${ }^{-1}$ día $^{-1}$ con 0 y 4, kg de MS de MFP, a 43,8 Mcal vaca $^{-1}$ día $^{-1}$ con $8 \mathrm{~kg}$ de MS de MFP, respectivamente. De acuerdo a este consumo de energía y a la energía en leche (NRC, 2001), se esperaba una producción de leche de al menos 16 litros vaca ${ }^{1} d^{1}$. Sin embargo, no fue así, por las razones antes discutidas; además pudieron estar afectando otros factores que no son considerados, como las características propias del animal, alimento y ambiente, los cuales interactúan entre sí, influyendo así en el consumo de alimento y la respuesta productiva del animal.

Por otro lado, aún cuando el concentrado ofrecido se formuló de tal manera que la dieta total tuviera $17 \%$ de $\mathrm{PB}$, el resultado obtenido por el programa del NRC (2001), muestra que las dietas totales (MFP + forraje + concentrado) tenían 22,2, 22,1 y $19,8 \%$ de $\mathrm{PB}$, con 0,4 , y $8 \mathrm{~kg}$ de materia seca de MFP, lo cual es superior al porcentaje de proteína recomendado para vacas Holstein, que es de $14 \%$, para vacas con 90 días en lactancia y produciendo $25 \mathrm{~kg}$ de leche al día (NRC, 2001). En dietas con alto contenido de proteína, la excreción de nitrógeno es alta, especialmente por vía urinaria, el cual se convierte en un potente contaminante del aire y del agua. Esto indica que aún cuando se menciona que hay mayor producción de leche con porcentajes de proteína más altos, su consumo en exceso, reduce su eficiencia de utilización, ya que hay más proteína de la que el animal fisiológicamente puede utilizar (Mulligan et al., 2004). Y esto pudo influir en los resultados aquí reportados.

\section{Carga animal y PRODUCCIÓN DE LECHE POR HECTÁREA \\ El consumo de MFP como suplemento} trajo como consecuencia disminución en el consumo de forraje de la pradera $y$, por tanto, un incremento significativo $(p<0,0001)$ en la carga animal (tabla III). Con el aumento de la carga animal, aún cuando la producción individual de leche no cambió, se incrementó la producción de leche por hectárea con el nivel de maíz fresco picado, aumentando más de 25 y $56 \mathrm{~kg}$ de leche ha- ${ }^{-1}$ cuando se ofrecieron 4 y $8 \mathrm{~kg}$ de materia seca vaca $^{-1}$ día $^{-1}$, respectivamente. Este incremento en la producción por hectárea por efecto de la carga animal es reportado por otros 


\section{MAÍZ FRESCO PICADO COMO SUPLEMENTO A VACAS LECHERAS}

Tabla III. Medias de consumo de materia seca, producción y composición de la leche, y carga animal de vacas Holstein en pastoreo con acceso a diferentes niveles de maíz fresco picado. (Average dry matter intake, milk production and composition, and stocking of dairy cows on pasture with access to different levels of fresh corn chopped).

\begin{tabular}{|c|c|c|c|c|}
\hline \multirow{2}{*}{\multicolumn{5}{|c|}{$\begin{array}{l}\text { Maíz fresco picado animal- }{ }^{-1} \mathrm{~d}^{-1}(\mathrm{~kg} \text { de MS) } \\
\text { Consumo de MS, } \mathrm{kg}^{-1} \text { animal }^{-1} \mathrm{~d}^{-1}\end{array}$}} \\
\hline & & & & \\
\hline Concentrado & 3,8 & 3,8 & 3,8 & \\
\hline Forraje & $8,4^{\mathrm{a}}$ & $5,2^{\mathrm{b}}$ & $3,1^{c}$ & 0,34 \\
\hline Total & $12.2^{\mathrm{a}}$ & $13,0^{\mathrm{a}}$ & $14,8^{b}$ & 0,36 \\
\hline $\begin{array}{l}\text { Producción de leche individual, } \mathrm{kg}_{\text {animal }}{ }^{-1} \mathrm{~d}^{-1} \\
\text { Composición de la leche, \% }\end{array}$ & 10,2 & 10,4 & 10,4 & 0,79 \\
\hline Grasa & 3,3 & 3,2 & 3,2 & 0,10 \\
\hline Proteína & 3,3 & 3,3 & 3,3 & 0,55 \\
\hline Lactosa & 4,2 & 4,4 & 4,3 & 0,89 \\
\hline Carga animal, vacas ha ${ }^{-1}$ & $3,8^{\mathrm{a}}$ & $6,2^{\mathrm{b}}$ & $8,8^{c}$ & 0,53 \\
\hline Producción de leche por hectárea, $\mathrm{kg} \mathrm{ha}^{-1} \mathrm{~d}^{-1}$ & $39,3^{\mathrm{a}}$ & $64,5^{\mathrm{b}}$ & $95,5^{c}$ & 6,61 \\
\hline
\end{tabular}

a,b,c Letras diferentes en una fila indican diferencias estadísticas ( $p<0,0001)$; MS: materia seca; EE: Error estándar.

autores (Améndola, 2002). Por su parte y utilizando un suplemento concentrado, Fike et al. (2003) mencionan que la producción de leche por hectárea se incrementa $61 \%$ con solo aumentar de 7,5 a 10 la carga animal, y que ésta puede ser igual a 10 cuando el forraje tiene un máximo crecimiento y la producción individual de leche es moderada. En este estudio se encontró una relación lineal entre maíz fresco picado y producción de leche por hectárea, incre-mentando $\left(\mathrm{R}^{2}=\right.$ $0,99) 0,75 \mathrm{~kg}$ de leche por $\mathrm{kg}$ de materia seca de maíz fresco picado ofrecido, resultado similar a $0,79 \mathrm{~kg}$ reportado por Améndola (2002), usando ensilado de maíz, o $0,80 \mathrm{~kg}$ reportado por Fike et al., (2003) usando concentrado. Esto indica que, independientemente de los efectos en la producción individual de leche, el uso de suplementos incrementa la producción de leche por hectárea.

Es pertinente enfatizar que la carga animal también tuvo una relación lineal con la cantidad de maíz fresco picado, al aumentar $\left(\mathrm{R}^{2}=0,99\right) 0,63$ vacas $^{-1} \mathrm{ha}^{-1}$ por cada $\mathrm{kg}$ de materia seca de maíz fresco picado ofrecido, valor superior a lo obtenido por Améndola (2000), quien reporta que la carga animal aumenta 0,4 vaca- $^{-1} \mathrm{ha}^{-1}$ por kg de MS de ensilado de maíz consumido; sin embargo, la carga animal que él reporta $(2,10$ y 3,61 vacas ha $^{-1}$, con 0 y 4,8 $\mathrm{kg}$ de materia seca de ensilado de maíz) es considerablemente menor a la de este trabajo con MFP.

\section{CONCLUSIONES}

La suplementación con MFP no mejoró ni en la producción individual ni la composición de la leche, pero aumentó el consumo total de materia seca y disminuyó de la pradera. Suplementar con MFP incrementa considerablemente la carga animal y por tanto la producción de leche por hectárea, aún cuando la tasa de sustitución fue moderada.

\section{AGRADECIMIENTOS}

Al Consejo Nacional de Ciencia y Tecnología (CONACyT)-México por la beca para realizar los estudios de Maestría, y a la Universidad Autónoma ChapingoMexico, a través del Módulo de Producción de Leche en Pastoreo por el apoyo a la realización de esta investigación. 


\section{RAMÍREZ, HERNÁNDEZ, AMÉNDOLA, MENDOZA, RAMÍREZY BURGUEÑO}

\section{BIBLIOGRAFÍA}

AOAC. 1984. Official Methods of Analysis. $5^{\mathrm{a}}$ ed. Association of Official Analytical Chemists. Washington. DC. EE.UU.

Améndola, M.R. 2002. A dairy system based on forages and grazing in temperate México. Animal Science Department. Chapingo University México.

Bargo, F., Muller, L.D., Delahoy, J.E. and Cassidy, T.W. 2002. Milk response to concentrate supplementation of high producing dairy cows grazing at two pasture allowance. J. Dairy Sci., 85: 1777-1792.

Bargo, F., Muller, L.D., Kolver, E.S. and Delahoy, J.E. 2003. Invited review: Production and digestion of supplemented dairy cows on pasture. J. Dairy Sci., 86: 1-42.

Church, D.C. 1988. The ruminant animal digestive physiology and nutrition. Prentice Hall. Englewood Clifs. New Jersey. USA. 54 pp.

Church, D.C., Pond, W.G. y Pond, K.R. 2003. Fundamentos de nutrición y alimentación de animales. Editorial Limusa. México. 334 pp.

Conchran, W.G., Autrey, K.M. and Cannon, C.Y. 1941. A double change over designs for dairy cattle feeding experiments. J. Dairy Sci., 24: 937-942.

Cunningham, G.J. 1999. Fisiología veterinaria. $2^{\mathrm{a}}$ ed. McGraw-Hill Interamericana. México.

Darby, H.M. and Lauer, J.G. 2002. Harvest date and hybrid influence on corn forage yield, quality, and preservation. Agron. J., 94: 559-566.

Delahoy, E.J., Muller, L.D., Bargo, F. T., Cassidy, W. and Holden, L.A. 2003. Supplemental carbohydrate sources for lactating dairy cows on pasture. J. Dairy Sci., 86: 906-915.

Fike, J.H., Staples, C.R., Sollenberger, L.E., Moore, J.E. and Head, H.H. 2002. Southeastern pasturebased dairy systems: housing, posilac, and supplemental silage effects on cow performance. J. Dairy Sci., 85: 866-878.

Fike, J.H., Staples, C.R., Sollenberger, L.E., Macoon, B. and Moore, J.E. 2003. Pasture forages, supplementation rate, and stocking rate; effects on dairy cow performance. J. Dairy Sci., 86: 1268-1281.

García, E. 1988. Modificaciones al sistema de clasificación climática de Koppen. México. 217 pp.
Geerken, C.M., Calzadilla, D. y González, R. 1987. Aplicación de la técnica de dos marcadores para medir el consumo de pasto y la digestibilidad de la ración de vacas en pastoreo suplementadas con concentrado. Pastos y forrajes, 10: 266-273.

Goering, H.R. and Van Soest. 1970. Forage fiber analysis. Departament of Agriculture Handbooks. Agricultural Research Service. USA.

Hernandez-Mendo, O. and Leaver D. 2004. Effect of replacing time available for grazing with time available for eating maize silage and soyabean meal on milk yield and feeding behaviour in dairy cows. Grass For. Sci., 59: 318-330.

Hernandez-Mendo, O. and Leaver J.D. 2006. Production and behavioural responses of highand low-yielding dairy cows to different periods of access to grazing or to a maize silage/ soyabean meal diet fed indoors. Grass For. Sci., 61: 335-346.

Holden, L.A., Muller, L.D., Lykos, T. and Cassidy, T.W. 1995. Effect of corn silage supplementation on intake and milk production in cows grazing pasture. J. Dairy Sci., 78: 154-160.

Ingvartsen, K.L. and Andersen, J.B. 2000. Integration of metabolism and intake regulation: a review focusing on periparturient animals. $J$. Dairy Sci., 83: 1573-1597.

Kolver, S.E. and Muller, L.D. 1998. Performance and nutrient intake of high producing Holstein cows consuming pasture or total mixed ration. J. Dairy Sci., 81: 1403-1411.

NRC. 2001. Nutrient requeriment of dairy cattle. $7^{\text {th }}$ revised edition. Nacional Research Council. Nacional Academy Press. Washington D.C.

Mulligan, F.J., Dillon, P., Callan, J.J., Rath, M. and O'Mara, F.P. 2004. Supplementary concentrate type affects nitrogen excretion of grazing dairy cows. J. Dairy Sci., 87: 3451-3460.

Pulido, R.G., Espindola, S., Laverne, A. y Uribe, H. 2002. Suplementación con maíz molido o roleado al vapor y comportamiento productivo de vacas lecheras en pastoreo primaveral. Arch. Zootec., 51: 397-400.

Roche, J.R. 2003. Effect of pregnancy on milk production and body weight from identical twin study. J. Dairy Sci., 86: 777-783.

Ruiz, T.M., Bernal, E. and Staples, C.R. 1995. 


\section{MAÍZ FRESCO PICADO COMO SUPLEMENTO A VACAS LECHERAS}

Effect of dietary neutral detergent fiber concentration and forage source on performance of lactanting cows. J. Dairy Sci., 78: 305-319.

SAS Institute. 1999. User's Guide: Statistics. $8^{\text {th }}$ ed. Inst. Inc. Cary, USA.

Sollenberger, L.E., Moore, J.E., Allen, V.G. and Pedreira, C.G.S. 2005. Reporting forage allowance in grazing experiments. Crop. Sci., 45: 896-900.

Tilley, J.M. and Terry, R.A. 1963. A two-stage technique for the in vitro digestion of forages crops. J. Br. Grassld. Soc., 18: 104-111.
Vazquez, O.P. and Smith, T.R. 2000. Factors affecting pasture intake and total dry matter intake in grazing dairy cows. J. Dairy Sci., 83: 2301-2309.

Wilkerson, A.V., Glenn, B.P. and McLeod, K.R. 1997. Energy and nitrogen balance in lactanting cows fed diets containing dry or high moisture corn either rolled or ground form. J. Dairy Sci., 80: 2487-2496.

Williams, C.H. 1962. The determination of chromic oxide in feces samples by atomic absorption spectrophotometers. J. Agric. Sci., 59: 381385. 